\title{
An Update on Survey Measures of Web-Oriented Digital Literacy
}

Social Science Computer Review Volume XX Number X Month XXXX xx-xx (C) 2008 Sage Publications $10.1177 / 0894439308318213$ http://ssc.sagepub.com hosted at http://online.sagepub.com

\section{Eszter Hargittai}

Northwestern University

\begin{abstract}
This article presents a test of a recently developed method for studying people's digital literacy. Self-reported instruments pose a risk of misreporting by respondents. Participants in the study are presented with items that are used to construct a previously established digital literacy measure, interspersed with strategically developed bogus items, to test how the latter measure up against the former. The bogus terms score the least in comparison with other items suggesting that the majority of people do not make up their responses to these questions, thereby adding support to the utility of the formerly proposed instrument. Results also show a considerable discrepancy between familiarity of older Internet-related terms and newer web-based concepts offering a more nuanced instrument for studies of users' digital literacy.
\end{abstract}

Keywords: digital literacy; Internet; method; measurement; self-efficacy; skill; survey; web

\section{Proxy Measures of Digital Literacy}

Most work looking at people's digital literacy relies on self-perceived skill measures to assess people's online abilities. Often, people's abilities are measured using the concept of self-efficacy. Bandura (1995) proposed this notion understood as "one's capability to organize and execute the courses of action required to manage prospective situations" (p. 2), a concept that has spurred much research in the field of psychology subsequently adopted by researchers studying computer and Internet uses (e.g., Corston \& Colman, 1996; Eastin \& LaRose, 2000; Torkzadeh \& Van Dyke, 2002; Whitley, 1997). Although the original idea may not have been to offer a proxy for actual skill, researchers studying computer use have often relied on such measures for this purpose.

Self-efficacy can be a helpful concept to understand how people approach various tasks, including the use of information and communication technologies; however, research has found that it is not the best proxy for people's actual digital literacy (Hargittai, 2005). In a study looking at the relationship between actual skill measures, based on people's online

\footnotetext{
Author's Note: The author is grateful to Jeremy Freese, Peter Miller, W. Russell Neuman, and Daniel O'Keefe for their helpful comments on the project. She also thanks the following people for their assistance with data collection and entry: Waleeta Canon, Gina Walejko, Soo An, Dan Li, and the group of undergraduate research assistants in the Web Use Project lab during the 2006-07 academic year. She acknowledges the generous support of the John D. and Catherine T. MacArthur Foundation, the Center for Advanced Study in the Behavioral Sciences, The Lenore Annenberg and Wallis Annenberg Fellowship in Communication, and Northwestern University's School of Communication Innovation Fund as well as its University Research Grants Committee.
} 
actions, Hargittai found that self-perceived skill is a less optimal predictor of actual skill than other items. For instance, asking people to self-rate their level of understanding of various computer and Internet-related terms on a 5-point scale is a stronger predictor of how well they are able to navigate online content compared with asking people how they think they can use the Internet.

Digital media are constantly evolving, however, which requires that the items proposed by any study be updated as new services are developed and become part of people's Internet-user repertoires. Although we know that the proposed 7-item index worked well as a proxy for actual user skill, can we conclude that additional terms would also work well to represent people's level of know-how about the Internet? The goal of this project was to test the performance of some new terms introduced to the index scale.

\section{Use of Bogus Items to Test the Instrument}

Survey methodology has always been plagued by the potential of respondents to misrepresent their knowledge and opinions. Philip Converse $(1964,1970)$ drew attention to these issues as early as the 1960s discussing the difficulty in measuring people's political opinions due to respondents' tendency to avoid "don't know" responses considered as "confessions of mental incapacity" (Converse, 1970, p. 170). In fact, decades before the writing of Converse, Hartley (1946) had already found that considerable numbers of college students would report attitudes about nonexistent national groups, again suggesting that selfreported items are not always a reflection of real beliefs. Studies such as these suggest that we have to be careful about survey construction, especially regarding items that ask people to report on opinions, attitudes, and know-how.

To test whether survey items used to construct the index measure of digital literacy are not simply checked off by respondents in a haphazard manner, three bogus items were included on a list of Internet-related terms used to measure the Internet-user abilities of the respondents (Hargittai, 2005) to see how the made-up entries would perform compared with real ones. Three types of bogus items were included on the survey.

- One item made up of terms resembling the names of actual computer and Internet-related concepts (proxy and pod) but a term that in its entirety does not mean anything: proxypod.

- An acronym given the prevalence of acronyms in the online world and among real terms: $J F W{ }^{1}$

- A term that was completely made up: filtibly.

The full list of items (27 real and 3 bogus) based on previous work that validated the instrument by correlating the measures with actual skill (Hargittai, 2005) was split into two lists so as not to overwhelm the respondent with too many items on one question. Two of the bogus items were included in the first batch of terms (proxypod and JFW), whereas the third (filtibly) was included as part of the second batch.

Respondents were presented with the following question and instruction:

How familiar are you with the following computer and Internet-related items? Please choose a number between 1 and 5, where 1 represents no understanding and 5 represents full understanding of the item. 
The order of the items on the two lists-constant on all surveys-was as follows (with the bogus items in italics).

First list: JPEG, frames, preference settings, newsgroups, PDF, refresh/ reload, advanced search, proxypod, weblog, $J F W$, bookmark, bookmarklet, spyware, bcc (on e-mail), and blog

Second list: tagging, tabbed browsing, RSS, wiki, malware, social bookmarking, podcasting, phishing, web feeds, firewall, filtibly, cache, widget, favorites, and torrent

The main focus of the overall survey was students' digital media uses, skills, and participation. These two digital literacy measures comprised a very small portion of the entire questionnaire and were located on p. 5 and p. 11, respectively, of the 23-page survey.

\section{Data and Methods}

A paper-and-pencil survey was administered to students enrolled in the only required course on the campus of an urban public research university in February-March, $2007 .^{2}$ Ninety-eight percent of all course sections ( 83 of 85) participated in the study with $82 \%$ of enrolled students in the course taking the survey. Given the high response rate and that this is the one course required of all students at this university, the sample is representative of the school's first-year student body.

The survey included an attentiveness question to see whether respondents were paying attention to the survey as a whole. The question was included midway through the survey and was worded as follows with the answer options below.

The purpose of this question is to assess your attentiveness to question wording. For this question please mark the Very often response.

\begin{tabular}{|lcccc|}
\hline$\square$ & $\square$ & $\square$ & $\square$ & $\square$ \\
Never & Rarely & Sometimes & Often & Very often \\
\hline
\end{tabular}

This question was surrounded by items to which very often was the least likely response (less than $2 \%$ marked very often to both the question preceding this one and the question following it). More than $96.5 \%$ marked very often in response to the attentiveness item. The $3.5 \%$ who did not choose this response are likely unreliable respondents and thus have to be included in the analyses with caution. It turns out, however, that such people's responses differ very slightly from other people's responses to the variables of interest in this article; therefore, the main discussion that follows is on the reduced sample of 1,189 respondents as opposed to the full sample of 1,247 respondents. Because nonattentiveness to survey questions is the focus of this piece, these cases are still included in the following discussion.

To test the performance of the survey instrument segments, I compare the average rating of all items with a focus on how the mean score of the bogus items matched up to the evaluations of the real items. If the bogus items scored lower than the real items then it is reasonable to assume that people do not select their responses randomly and that the 
Table 1

Average Score (on a 1 to 5 Point Scale) of

Internet-Related Items Asked on the First Question

\begin{tabular}{lc}
\hline Term & Mean \\
\hline Reload & 4.68 \\
Advanced search & 4.44 \\
Bookmark & 4.36 \\
Spyware & 4.09 \\
Preference setting & 4.03 \\
Blog & 3.97 \\
PDF & 3.62 \\
JPG & 3.44 \\
Weblog & 3.25 \\
Newsgroup & 2.94 \\
Frames & 2.79 \\
Bcc (on e-mail) & 2.47 \\
Bookmarklet & 2.34 \\
Proxypod & 1.87 \\
JFW & 1.60 \\
\hline
\end{tabular}

Note: The bogus items are indicated in italics.

instrument measures people's understanding of Internet-related terms. However, if the bogus items perform significantly better than or as well as many of the real items then we must question the validity of the measure as a proxy for digital literacy because it suggests that respondents are not reporting levels of understanding if they claim to understand nonexistent terms (i.e., proxypod, JFW, and filtibly).

\section{Findings}

Tables 1 to 3 present the mean rating score of the 30 items included on the two questions. Table 1 presents the first batch of 15 items in the decreasing order of understanding, Table 2 shows the ratings of the second batch of items, whereas Table 3 aggregates all of the items into one list in overall decreasing rank order. Significant literature exists about the order and context effects on questionnaires (e.g., Schuman \& Presser, 1981) and thus the decision to show the results separately here by the group of items in the manner they were presented to respondents.

In both Tables 2 and Table 3, we see that the bogus items scored lower than any other items on the list. Although not everybody marked the bogus items as 1 indicating no understanding, overall these items are the lowest-ranked in comparison with other items on the same list of terms. Also, looking at the distribution of responses by comparing histograms of every item (Figure 1), it is clear that the bogus items display the most unimodal distribution of all terms. That is, on some other terms that are, on average, rated as barely understood, the most savvy users still report high levels of understanding. However, bogus items 
Table 2

Average Score (on a 1 to 5 Point Scale)

of Internet-Related Items Included on the Second Question

\begin{tabular}{lc}
\hline Term & Mean \\
\hline Favorites & 4.42 \\
Firewall & 3.85 \\
Tagging & 3.30 \\
Tabbed browsing & 2.78 \\
Podcasting & 2.71 \\
Web feeds & 2.55 \\
Torrent & 2.40 \\
Cache & 2.29 \\
Wiki & 2.29 \\
Phishing & 1.80 \\
Widget & 1.78 \\
Malware & 1.75 \\
Social bookmarking & 1.68 \\
RSS & 1.61 \\
Filtibly & 1.34 \\
\hline
\end{tabular}

Note: The bogus item is indicated in italics.

are the least likely to get high scores. One can eyeball all this by looking at the height of the rightmost column on each graph representing the proportion of respondents, who rated their understanding of the respective items with the highest score of 5. For bogus items, we do not observe bumps for this rating on the histograms unlike the case of real items.

It is also noteworthy to compare the relative rankings of the three bogus terms. The bogus item, filtibly, with absolutely no relation to a real item scored the lowest. The acronym JFW was the second lowest while drawing on existing terms (proxy and pod yielding proxypod) for an item may confuse people into thinking that they know about it. Thus, when constructing bogus items for the purposes of testing survey instruments, it is important to take care in picking the make-believe terms so as to guarantee their truly bogus nature.

Interestingly, on the aggregated list of items, there are some terms from the second list that score lower than one of the bogus items from the first list, although the differences are minimal with differences as little as 0.07 (between proxypod and phishing) and no more than 0.26 (between proxypod and RSS). This suggests that context of the items matters to some extent. That is, while within a certain group a bogus item may be scored the lowest, there may be an overall effect of placement on the instrument as to various items' absolute ratings. The implication of this finding is that we must be careful about comparing relative ratings across questions although given the overall ranking, these context effects seem only minimal.

As noted earlier, problem cases were excluded from the analyses. A look at the average scores with the problem cases included yields very similar results (available from the author upon request). Although the relative order of a few real items changes (at most a switch between two terms), the placement of the bogus terms in the rank order is robust. 
Table 3

Average Score (on a 1 to 5 Point Scale)

of All Internet-Related Items Included on the Two Questions

\begin{tabular}{lll}
\hline Question & \multicolumn{1}{c}{ Term } & Mean \\
\hline 1 & Reload & 4.68 \\
1 & Advanced search & 4.44 \\
2 & Favorites & 4.42 \\
1 & Bookmark & 4.36 \\
1 & Spyware & 4.09 \\
1 & Preference setting & 4.03 \\
1 & Blog & 3.97 \\
2 & Firewall & 3.85 \\
1 & PDF & 3.62 \\
1 & JPG & 3.44 \\
2 & Tagging & 3.30 \\
1 & Weblog & 3.25 \\
1 & Newsgroup & 2.94 \\
1 & Frames & 2.79 \\
2 & Tabbed browsing & 2.78 \\
2 & Podcasting & 2.71 \\
2 & Web feeds & 2.55 \\
1 & Bcc (on e-mail) & 2.47 \\
2 & Torrent & 2.40 \\
1 & Bookmarklet & 2.34 \\
2 & Cache & 2.29 \\
2 & Wiki & 2.29 \\
1 & Proxypod & 1.87 \\
2 & Phishing & 1.80 \\
2 & Widget & 1.78 \\
2 & Malware & 1.75 \\
2 & Social bookmarking & 1.68 \\
2 & RSS & 1.61 \\
1 & JFW & 1.60 \\
2 & Filtibly & 1.34 \\
\hline & & \\
\hline & &
\end{tabular}

Note: Normal text indicates items from the first batch, boldface indicates items from the second batch, and the three bogus items are indicated in italics.

Moreover, most differences are at most 0.02 with just one case of 0.03 difference between the average score for all respondents versus the problem cases excluded.

\section{Conclusion}

Previous work has found that asking people to rate their level of understanding of Internet-related terms serves as a better proxy for actual online skills than some other traditional measures such as self-perceived skill (Hargittai, 2005). Owing to the ever-evolving 
Figure 1
Distribution of Responses to Each Item, Presented in Decreasing Order (Starting in Upper Left Corner) of Average Score With the Bogus Items Presented Last With Gray Background
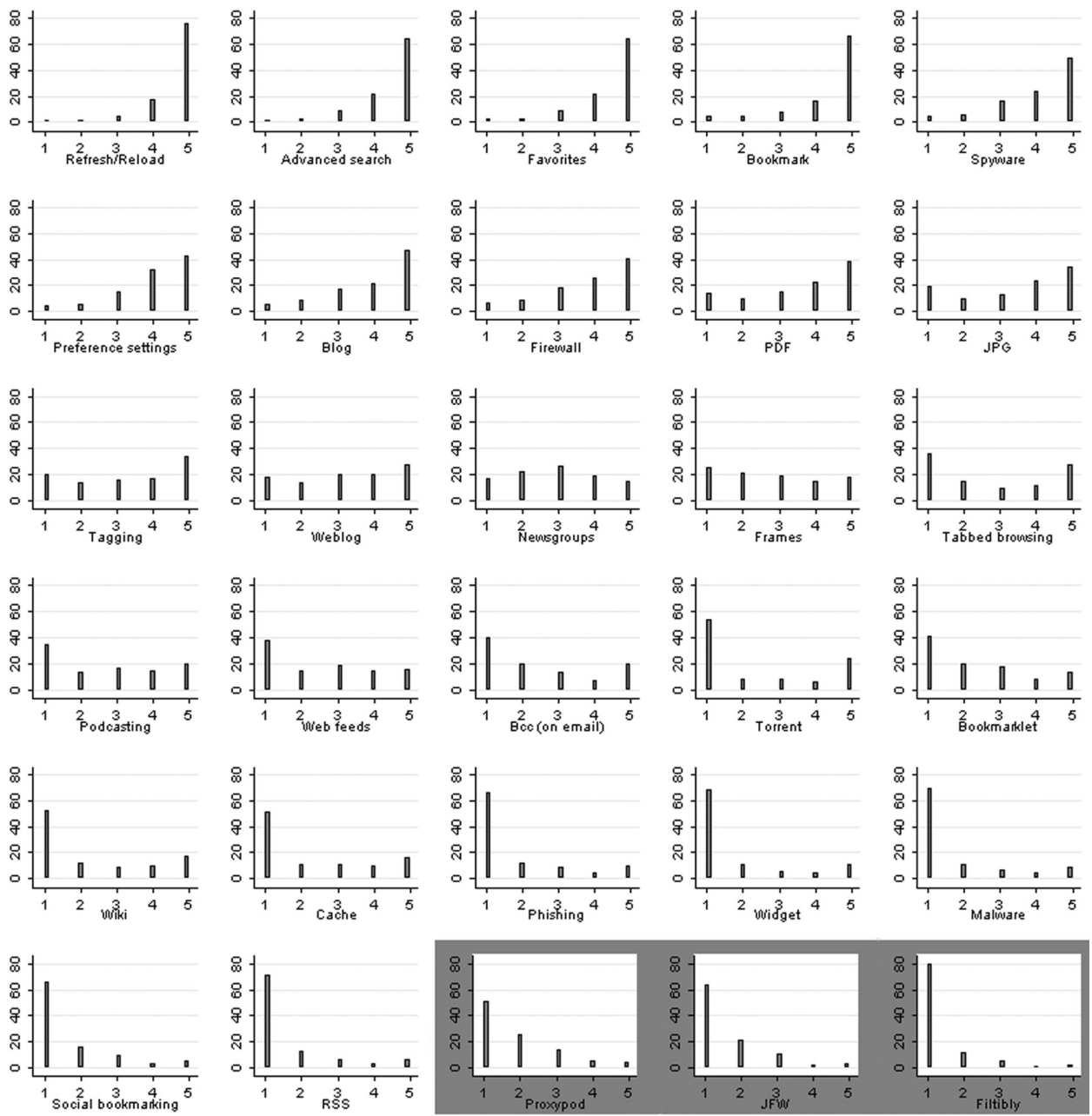

nature of digital media, items making up constructs of digital literacy may become outdated or may not capture the necessary nuances in differences among people over time. This change in the medium requires that we update such instruments. The goal of this study was to test the robustness of survey items that offer an update to a previously established index measure of online ability. Given that the bogus items included in the study were rated as the lowest when compared to other items on the list, we can conclude that self-reported understanding of Internet-related items is not plagued by haphazard responses on the part of survey participants. 


\section{Notes}

1. Although the acronym JFW does have various instances on the web, none of these seems to be widely read resources. There are no unregistered three-letter domain names suggesting that it would be nearly impossible to pick an acronym that will have absolutely no representation online. Because students took this survey on paper, chances are miniscule that they would recognize an actual site behind the acronym.

2. The author of this article has never been formally affiliated with this institution. The campus was not picked because of convenience, but rather, owing to its population's relevance for the main questions of the overall research project having to do with digital media uses and social inequality.

\section{References}

Bandura, A. 1995. Self-efficacy in changing societies. New York: Cambridge University Press

Converse, P. E. (1964). The nature of belief systems in mass publics. In D. E. Apter (Ed.), Ideology and discontent (pp. 206-261). London: The Free Press of Glencoe.

Converse, P. E. (1970). Attitudes and non-attitudes: Continuation of a dialogue. In E. R. Tufte (Ed.), A quantitative analysis of social problems (pp. 168-189). Reading, MA: Addison-Wesley.

Corston, R., \& Colman, A. (1996). Gender and social facilitation effects on computer competence and attitudes towards computers. Journal of Educational Computing Research, 14, 171-183.

Eastin, M. A, \& LaRose, R. L (2000). Internet self-efficacy and the psychology of the digital divide. Journal of Computer-Mediated Communication, 6(1).

Hargittai, E. (2005). Survey measures of web-oriented digital literacy. Social Science Computer Review, 23, 371-379.

Hartley, E. L. (1946). Problems in prejudice. New York: Kings Cross Press.

Schuman, H., \& Presser, S. (1981). Questions and answers in attitude surveys. New York: Academic Press.

Torkzadeh, G., \& Van Dyke, T. (2002). Effects of training on internet self-efficacy and computer user attitudes. Computers in Human Behavior, 18, 479-494.

Whitley, B. (1997). Gender differences in computer-related attitudes and behavior: A meta-analysis. Computers in Human Behavior, 13, 1-22.

Eszter Hargittai (PhD, Sociology, Princeton) is on the faculty in the Communication Studies Department at Northwestern University, where she heads the Web Use Project. She spent the 2006-07 academic year as a fellow at the Center for Advanced Study in the Behavioral Sciences. Her research focuses on the social and policy implications of information technologies with a particular interest in how information technologies may contribute to or alleviate social inequalities. Her research projects have looked at differences in people's webuse skills, the evolution of search engines, the organization and presentation of online content, political uses of information technologies, and how information technologies are influencing the types of cultural products people consume. She can be reached at sscore08@webuse.org. 